\title{
Morocco's First Biobank: Establishment, Ethical Issues, Biomedical Research Opportunities, and Challenges
}

\author{
Saida Lhousni, ${ }^{1}$ Karam Yahya Belmokhtar, ${ }^{1}$ Ihab Belmokhtar, ${ }^{1}$ Mounia Elidrissi Errahhali, ${ }^{1}$ \\ Manal Elidrissi Errahhali, ${ }^{1}$ Redouane Boulouiz, ${ }^{1,2}$ Mariam Tajir, ${ }^{3}$ Majida Charif, \\ Khawla Zerrouki, ${ }^{3}$ Noufissa Benajiba, ${ }^{5}$ Maria Rkain, ${ }^{5}$ Abdeladim Babakhouya, ${ }^{5}$ \\ Hatim Kouismi, ${ }^{6}$ Afaf Thouil, ${ }^{6}$ Hanane Latrach, ${ }^{7}$ Rim Amrani, ${ }^{8}$ Sahar Messaoudi, ${ }^{8}$ \\ Anass Ayyad, ${ }^{8}$ Zaina Sidqi, ${ }^{9}$ Khalid Andaloussi Serraj, ${ }^{10}$ Siham Hamaz, ${ }^{10}$ Habiba Alaoui, ${ }^{10}$ \\ Houda Bachir, ${ }^{10}$ Yassamine Bentata, ${ }^{11}$ Intissar Haddiya, ${ }^{11}$ Mohammed Choukri, ${ }^{12}$ \\ Rachid Seddik, ${ }^{1,13}$ Amal Bennani, ${ }^{14}$ Siham Dikhaye, ${ }^{15}$ Bouchra Oneib, ${ }^{16}$ \\ Fatima Elghazouani, ${ }^{16}$ Omar El Mahi, ${ }^{17}$ Adnane Benzirar, ${ }^{17}$ Ayat Allah Oufkir, ${ }^{18}$ \\ Brahim Housni, ${ }^{19}$ Ahmed Mimouni, ${ }^{20}$ Hanane Saadi, ${ }^{20}$ Mohammed Belahcen, ${ }^{21}$ \\ Tijani El Harroudi, ${ }^{22}$ Meryem Ouarzane, ${ }^{1,23}$ and Mohammed Bellaoui $\mathbb{D D}^{1}$
}

${ }^{1}$ Genetics Unit, Medical Sciences Research Laboratory, Faculty of Medicine and Pharmacy, University Mohammed Premier, Oujda, Morocco

${ }^{2}$ Higher Institute of Nursing Professions and Health Technologies, Oujda, Morocco

${ }^{3}$ Medical Genetics Unit, Central Laboratory, Mohammed VI University Hospital, Faculty of Medicine and Pharmacy, University Mohammed Premier, Oujda, Morocco

${ }^{4}$ Genetics, and Immuno-Cell Therapy Team, Faculty of Sciences, University Mohammed Premier, Oujda, Morocco

${ }^{5}$ Department of Pediatrics, Mohammed VI University Hospital, Faculty of Medicine and Pharmacy, University Mohammed Premier, Oujda, Morocco

${ }^{6}$ Department of Pulmonology, Mohammed VI University Hospital, Faculty of Medicine and Pharmacy, University Mohammed Premier, Oujda, Morocco

${ }^{7}$ Department of Endocrinology, Mohammed VI University Hospital, Faculty of Medicine and Pharmacy, University Mohammed Premier, Oujda, Morocco

${ }^{8}$ Department of Neonatology, Mohammed VI University Hospital, Faculty of Medicine and Pharmacy, University Mohammed Premier, Oujda, Morocco

${ }^{9}$ Transfusion Regional Centre, Oujda, Morocco

${ }^{10}$ Department of Internal Medicine, Mohammed VI University Hospital, Faculty of Medicine and Pharmacy, University Mohammed Premier, Oujda, Morocco

${ }^{11}$ Nephrology and Kidney Transplantation Unit, Mohammed VI Faculty of Medicine and Pharmacy, University Hospital, University Mohammed Premier, Oujda, Morocco

${ }^{12}$ Biochemistry Unit, Central Laboratory, Mohammed VI University Hospital, Faculty of Medicine and Pharmacy, University Mohammed Premier, Oujda, Morocco

${ }^{13}$ Hematology Unit, Central Laboratory, Mohammed VI University Hospital, Faculty of Medicine and Pharmacy, University Mohammed Premier, Oujda, Morocco

${ }^{14}$ Pathology Unit, Central Laboratory, Mohammed VI University Hospital, Faculty of Medicine and Pharmacy, University Mohammed Premier, Oujda, Morocco

${ }^{15}$ Department of Dermatology, Mohammed VI University Hospital, Faculty of Medicine and Pharmacy, University Mohammed Premier, Oujda, Morocco

${ }^{16}$ Department of Psychiatry, Mohammed VI University Hospital, Faculty of Medicine and Pharmacy, University Mohammed Premier, Oujda, Morocco

${ }^{17}$ Department of Vascular Surgery, Mohammed VI University Hospital, Faculty of Medicine and Pharmacy, University Mohammed Premier, Oujda, Morocco 


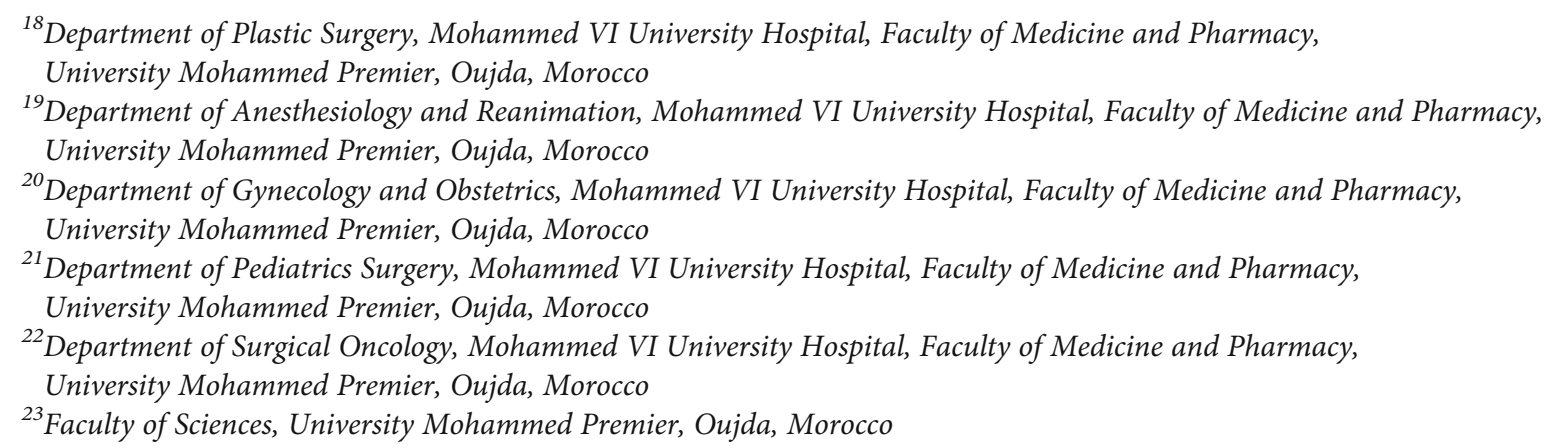

Correspondence should be addressed to Mohammed Bellaoui; bmbellaoui@gmail.com

Received 26 August 2020; Revised 15 November 2020; Accepted 18 November 2020; Published 8 December 2020

Academic Editor: Daniel Diaz

Copyright (c) 2020 Saida Lhousni et al. This is an open access article distributed under the Creative Commons Attribution License, which permits unrestricted use, distribution, and reproduction in any medium, provided the original work is properly cited.

Background. Biobanks are highly organized infrastructures that allow the storage of human biological specimens associated with donors' personal and clinical data. These infrastructures play a key role in the development of translational medical research. In this context, we launched, in November 2015, the first biobank in Morocco (BRO Biobank) in order to promote biomedical research and provide opportunities to include Moroccan and North African ethnic groups in international biomedical studies. Here, we present the setup and the sample characteristics of BRO Biobank. Methods. Patients were recruited at several departments of two major health-care centers in the city of Oujda. Healthy donors were enrolled during blood donation campaigns all over Eastern Morocco. From each participant, personal, clinical, and biomedical data were collected, and several biospecimens were stored. Standard operating procedures have been established in accordance with international guidelines on human biobanks. Results. Between November 2015 and July 2020, 2446 participants were recruited into the BRO Biobank, of whom 2013 were healthy donors, and 433 were patients. For healthy donors, the median age was 35 years with a range between 18 and 65 years and the consanguinity rate was $28.96 \%$. For patients, the median age was 11 years with a range between 1 day and 83 years. Among these patients, 55\% had rare diseases (hemoglobinopathies, intellectual disabilities, disorders of sex differentiation, myopathies, etc.), $13 \%$ had lung cancer, $4 \%$ suffered from hematological neoplasms, $3 \%$ were from the kidney transplantation project, and 25\% had unknown diagnoses. The BRO Biobank has collected 5092 biospecimens, including blood, white blood cells, plasma, serum, urine, frozen tissue, FFPE tissue, and nucleic acids. A sample quality control has been implemented and suggested that samples of the BRO Biobank are of high quality and therefore suitable for high-throughput nucleic acid analysis. Conclusions. The BRO Biobank is the largest sample collection in Morocco, and it is ready to provide samples to national and international research projects. Therefore, the BRO Biobank is a valuable resource for advancing translational medical research.

\section{Introduction}

A biobank is a highly organized structure that stores human biological samples associated with the donors' medical and personal information. The last several decades have seen a dynamic development of biobanks. Indeed, hundreds of biobanks have been created in many countries to provide thousands of human biospecimens from patients and/or healthy individuals to research programs. These resources have become indispensable tools for biomedical research [1, 2]. Indeed, biobanks have been used by researchers to advance treatment and prevention of many diseases and to develop innovative diagnostic tests [3-7].

Biobanking should harmonize a number of steps: collection of biological samples (tissues, blood, urine, etc.), processing, storage, and quality control of these samples; management of medical information; and finally, providing them to research projects. Therefore, biobanking requires coordinated action among many stakeholders such as donors, clinicians, nurses, laboratory technicians, scientists, healthcare providers, and ethicists [8].

A major challenge for biobanking is to ensure that the stored samples are well preserved and of high quality. Another challenge is to make these samples easily accessible to researchers leading to the generation of reliable research data accepted by the scientific community. Therefore, standard operating procedures (SOPs) describing all aspects of biobanking (sample collection, processing, storage, and quality control) have been established by various biobank organizations, such as the International Society for Biological and Environmental Repositories (ISBER), the National Cancer Institute (NCI), OECD Recommendation on Human Biobanks and Genetic Research Databases, and the European, Middle Eastern and African Society for Biobanking and Biopreservation (ESBB) [9-15].

Although biobanks have been in place in high-income countries for many years; it is only recently that they have been implemented in some low- and middle-income 
countries [16-19]. In this context, we launched in November 2015 a biobank in the eastern region of Morocco (BRO Biobank) in order to promote biomedical research in Morocco where research resources are limited. In this paper, we present all details of the creation of the BRO Biobank (ethical aspect, patient and healthy donor recruitment, processing, storage and quality control of the biospecimens, and management of medical information). We also present the sample collection of the BRO Biobank. We then discuss the challenges of the BRO Biobank.

\section{Materials and Methods}

2.1. Ethical Considerations. In Morocco, there is currently no specific law on biobanks or legislation addressing biomedical research. However, there is a law on the protection of individuals with regard to the processing of personal data [20]. Therefore, in accordance with this law, which is relevant for biomedical research and biobanking, we have established an ethical protocol for the BRO Biobank. We have also taken into account the laws and ethical guidelines for biobanks or biomedical research involving human subjects from different high-income countries such as Belgium, Finland, France, Iceland, and Germany [21-25].

The BRO Biobank's ethical protocol was approved by the Ethical Review Committee for Biomedical Research of the Faculty of Medicine and Pharmacy of Casablanca (CERBC). The authorization for personal data processing was obtained from the data protection agency of Morocco (CNDP). The main ethical issues addressed in the BRO Biobank's ethical protocol were confidentiality, informed consent, secondary use of samples and data over time, use of genetic material, children's participation in biobanks, return of results to participants, and sharing of data and samples with other scientists. Written informed consent was obtained from each participant at enrolment.

2.2. BRO Biobank's Facility. The BRO Biobank is located at the Genetics Unit of the Faculty of Medicine and Pharmacy of Oujda. The Genetics Unit is organized into a cytogenetic lab, a molecular biology lab, and a room with several freezers for storing samples.

2.3. Patients and Healthy Donors. Patients were recruited into the BRO Biobank at several medical and chirurgical departments of two major health care centers in the city of Oujda (Mohammed VI University Hospital Center and El Farabi Regional Hospital). Regarding healthy individuals, recruitment into the BRO Biobank was carried out at the Regional Blood Transfusion Center of Oujda and during the blood donation campaigns in the other cities of Eastern Morocco. Patients or healthy donors were approached and given explanations about the purpose of biobanking. They were then invited to participate in the BRO Biobank. Individuals who agreed to participate in the BRO Biobank were asked to sign an informed consent available in Arabic and French.

2.4. Collection, Transport, and Reception of Biospecimens at the BRO Biobank. Blood or tissue samples (surgical specimens or biopsies) were collected by health professionals in appropriate containers and transported to the BRO Biobank as quickly as possible in a cooled container by the BRO Biobank staff. At the BRO Biobank's facility, the samples were kept in the refrigerator until they were registered in the BRO Biobank. For each donor, a code was given to identify all types of his biological samples and a file was created to keep his signed informed consent and all his medical and personal information.

2.5. Processing of Tissue Samples. Tissue samples (surgical specimens or biopsies) were collected by healthcare professionals either in a tube containing the homemade stabilizing reagent "RNAlater" or an empty tube. They were then transported to the BRO Biobank's facility within less than one hour. Upon arrival, tissue samples were recorded in the BRO Biobank and then stored at $-80^{\circ} \mathrm{C}$.

2.6. Preparation of Plasma from Blood Samples. To prepare plasma, the blood samples were collected in EDTA tubes and then transported at $4^{\circ} \mathrm{C}$ to the BRO Biobank's facility. Within one hour of receiving the blood samples, the plasma was separated from the whole blood by centrifugation at $1800 \times \mathrm{g}$ for 15 minutes at $+4^{\circ} \mathrm{C}$. For each tube, after centrifugation, the upper phase, which corresponds to the plasma, was aspirated and aliquoted into labelled storage tubes (500 $\mu \mathrm{l} /$ tube) and stored at $-80^{\circ} \mathrm{C}$.

2.7. Preparation of Serum from Blood Samples. Blood samples intended for the preparation of serum were drawn in dry tubes. The tubes were inverted 8 times immediately after blood draw to ensure efficient coagulation and were then transported at room temperature to the BRO Biobank's facility. The tubes were centrifuged at $1500 \times \mathrm{g}$ for 15 minutes at room temperature, and then, the serum was aspirated and aliquoted into labelled storage tubes $(500 \mu \mathrm{l} /$ tube $)$ and stored at $-80^{\circ} \mathrm{C}$.

2.8. Preparation of White Blood Cells (WBC) from Blood Samples. To prepare WBC, blood samples were collected in EDTA tubes, transported at $+4^{\circ} \mathrm{C}$ to the BRO Biobank's facility, and processed within one hour of blood draw. Thus, after arrival to the BRO Biobank's facility, tubes were centrifuged at $1800 \times \mathrm{g}$ for 15 minutes at $+4^{\circ} \mathrm{C}$. For each tube, the buffy coat layer containing most of the WBC was aspirated and then transferred into $2 \mathrm{ml}$ tubes. To remove excess red blood cells, the WBCs were washed by adding 3 volumes of the TE buffer $(20: 5)$ and then centrifugation at $2800 \times \mathrm{g}$ for 15 minutes at $+4^{\circ} \mathrm{C}$. Then, 10 volumes of a homemade RNAlater reagent was added to the WBC and the mixture was incubated at $+4^{\circ} \mathrm{C}$ overnight. The following day, 1 volume of PBS was added and then, the tube was centrifuged at maximum speed $(5000 \mathrm{~g})$ for $10 \mathrm{~min}$ at room temperature. The supernatant was removed, and the WBCs were stored at $-80^{\circ} \mathrm{C}$.

2.9. DNA Extraction from Blood Samples. Blood samples intended for DNA extraction were drawn in EDTA tubes, kept at a temperature between $4^{\circ} \mathrm{C}$ and $25^{\circ} \mathrm{C}$, and processed within 24 hours of blood draw. In the case where the blood samples cannot be processed for DNA extraction within this period, they have been stored at $-20^{\circ} \mathrm{C}$ until use. DNA extraction was carried out using the phenol/chloroform method as 
described [26]. DNA was aliquoted into labeled storage tubes and stored at $-20^{\circ} \mathrm{C}$.

2.10. RNA Extraction from Blood Samples. To extract RNA, blood samples were collected in EDTA tubes and transported at $+4^{\circ} \mathrm{C}$ to the BRO Biobank's facility. The blood samples must be processed within one hour of blood draw, otherwise RNA will be rapidly degraded because they are very unstable. Thus, upon arrival at the BRO Biobank's facility, the tubes were immediately centrifuged at $1800 \times \mathrm{g}$ for 15 minutes at $+4^{\circ} \mathrm{C}$. For each tube, the buffy coat layer was aspirated and washed by adding 3 volumes of TE buffer $(20: 5)$ and then centrifugation at $2800 \times \mathrm{g}$ for 15 minutes at $+4^{\circ} \mathrm{C}$. Then, the pellet containing the WBCs was resuspended in $1 \mathrm{ml}$ of "TRizol" reagent and the RNAs were isolated using "TRizol" reagent (Invitrogen). If RNA extraction cannot be performed immediately, the mixture of WBC plus "TRizol" reagent can be stored at $-80^{\circ} \mathrm{C}$ for 15 days until use. After RNA isolation, RNA is aliquoted into labeled storage tubes and stored at $-80^{\circ} \mathrm{C}$.

\subsection{Quality Control Analysis of DNA Samples Preserved in} the BRO Biobank. BRO Biobank quality control was carried out every 6 months. 5\% of tissue samples and 5\% of DNA samples of the BRO Biobank were randomly selected and used to assess the DNA quality. Several methods have been used for quality control analysis of DNA samples stored in the BRO Biobank: The yield and purity of DNA samples were assessed by measuring the optical density $\left(\mathrm{OD}_{260} / \mathrm{OD}_{280}\right)$ ratio using NanoDrop spectrophotometry (Thermo Fisher Scientific). A DNA sample of good quality should have an $\mathrm{OD}_{260} / \mathrm{OD}_{280}$ ratio between 1.8 and $2.1[27,28]$. The DNA integrity was evaluated by $0.8 \%$ agarose gel electrophoresis, and a quality score was assigned for each DNA sample based on the $\mathrm{OD}_{260} / \mathrm{OD}_{280}$ ratio and the DNA electrophoresis profile as described in [28].

In addition, DNA quality was assessed by enzymatic digestion (HindIII enzyme, Thermo Fisher Scientific, Lithuania) followed by gel electrophoresis as described in $[29,30]$. DNA quality was also evaluated by PCR amplification of four different regions of the $\beta$-globin gene using the primers shown in Table 1. Obtaining four amplification bands was the indication of a good-quality DNA $[27,29,31]$.

2.12. Quality Control Analysis of RNA Samples Preserved in the BRO Biobank. $10 \%$ of tissue samples and $10 \%$ of RNA samples of the BRO Biobank were randomly selected and used for RNA quality assessment. As for DNA, the yield and purity of RNA samples were assessed by measuring the optical density $\left(\mathrm{OD}_{260} / \mathrm{OD}_{280}\right)$ ratio using NanoDrop spectrophotometry (Thermo Fisher Scientific). A RNA sample of good quality should have an $\mathrm{OD}_{260} / \mathrm{OD}_{280}$ ratio $<1.8$ [28]. The RNA integrity was evaluated by $1.5 \%$ nondenaturing agarose gel electrophoresis followed by analysis of the $28 \mathrm{~S}$ and $18 \mathrm{~S}$ ribosomal RNAs [27]. RT-PCR was also used to evaluate the RNA integrity as described [32]. In this assay, a region of the GAPDH (glyceraldehyde-3-phosphate dehydrogenase) gene was amplified using the following primers: GAPDH forward: $5^{\prime}$-CCCATGTTCGTCATGGGTGT-3 ${ }^{\prime}$
TABLE 1: List of primer sequences used for PCR analysis of the $\beta$ globin gene.

\begin{tabular}{lcc}
\hline $\begin{array}{l}\text { Primer } \\
\text { name }\end{array}$ & Sequence $\left(5^{\prime} \longrightarrow 3^{\prime}\right)$ & $\begin{array}{c}\text { Size of the PCR } \\
\text { product in bp }\end{array}$ \\
\hline GH20 & GAAGAGCCAAGGACAGGTAC & 268 \\
PC04 & CAACTTCATCCACGTTCACC & \\
RS42 & GCTCACTCAGTGTGGCAAAG & 536 \\
KM29 & GGTTGGCCAATCTACTCCCAGG & \\
RS40 & ATTTTCCCACCCTTAGGCTG & 989 \\
RS80 & TGGTAGCTGGATTGTAGCTG & \\
KM29 & GGTTGGCCAATCTACTCCCAGG & 1327 \\
RS80 & TGGTAGCTGGATTGTAGCTG & \\
\hline
\end{tabular}

and GAPDH reverse: $5^{\prime}$-ATAGCACCTTCCTGAGTACTG GT-3'.

2.13. BRO Biobank Data Management. Three different Excel files were created for data management of the BRO Biobank: the first contains all of the donors' medical and personal information. This file is kept in a secure and locked location and only some laboratory members have access to it. The second file is a copy of the first one but does not contain names, medical record number, or any other information that could identify the donors. In this file, information on participants was made anonymous to protect their identity following the guidelines on research ethics. The third file contains information about all biological samples of the BRO Biobank such as the nature of the sample, concentration, volume, quality, and location of the sample in the freezer. As for the second file, code numbers are used instead of names for identification in this file.

2.14. Statistical Analysis. Data collection and calculations were performed with Microsoft Excel software version 2013. For qualitative variables, we calculated the percentage. Quantitative variables were expressed as mean and standard deviation (SD).

\section{Results}

A total of 2446 participants were recruited in the BRO Biobank in a period starting from November 2015 to July 2020. Of those participants, 2013 were individuals from the general population of Eastern Morocco "healthy participants" and 433 were patients. Below are the characteristics of these two groups and the corresponding samples stored in the BRO Biobank.

3.1. Characteristics of Healthy Participants Enrolled in the BRO Biobank. Of the healthy participants, 1063 were men and 950 were women, with a male-to-female ratio of 1.12. Participants' median age was 35 years with a range between 18 and 65 years, and the consanguinity rate was $28.96 \%$. Taking into account the percentage of the population living in each region in Eastern Morocco, 530 participants were recruited from Nador, followed, respectively, by Oujda (497), Berkane (241), Taourirt (224), Guercif (182), Figuig 


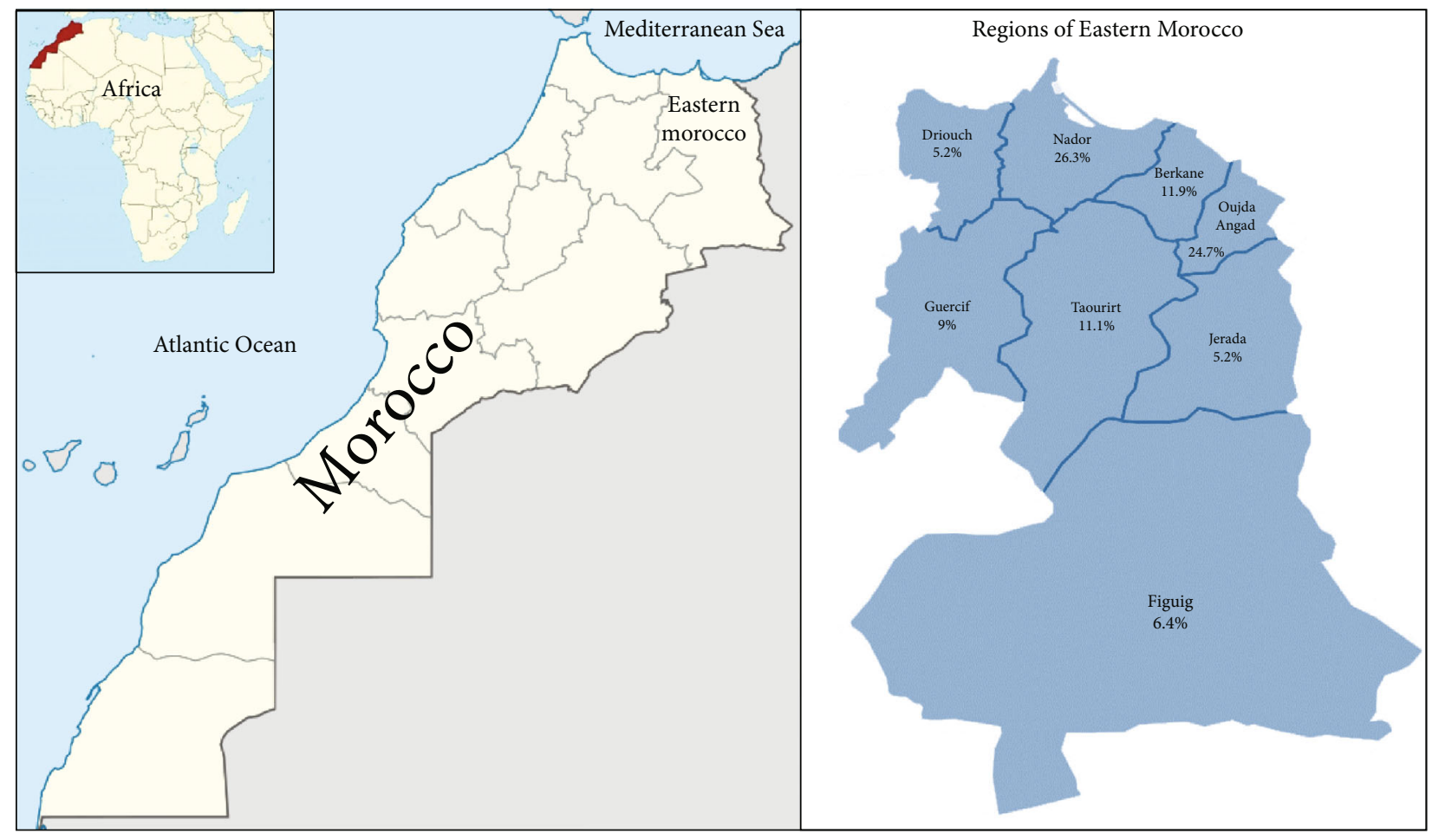

FIGURE 1: Schematic representation of Eastern Morocco region adapted from "Morocco Map Location" and "Regional Map of Morocco" [33, 34]. The distribution of healthy participants enrolled in the BRO Biobank by region of Eastern Morocco is shown.

(129), Jerada (105), and Driouch (105). The distribution of healthy participants of the BRO Biobank by the region of Eastern Morocco is shown in Figure 1. Biobanking from these participants has resulted in a total of 4341 biospecimens. Of these, 2013 were blood samples, 2013 serums, 261 DNA, and 54 white blood cells (Figure 2).

\subsection{Characteristics of Patients Enrolled in the BRO Biobank.} The personal information of the patients enrolled in the BRO Biobank is described in Table 2. Of the participants, 260 were men and 173 were women, with a male-to-female ratio of 1.50 . The median age of participants was 11 years (range 1 days-83 years). In terms of consanguinity, $47 \%$ of the patients were from a consanguineous marriage, and of these, $68 \%$ were from 1st-degree consanguinity, $20 \%$ from 2nd-degree consanguinity, and $12 \%$ from 3rd-degree consanguinity. A family history of genetic diseases was found in $36 \%$ of the patients. $62 \%$ of total cases were recruited in the BRO Biobank in the context of diagnosis and research; in contrast, $38 \%$ of cases were enrolled for research only.

The distribution of participants by referral department is illustrated in Figure 3. The majority of patients enrolled in the BRO Biobank were hospitalized in the department of pediatrics (41\%), followed by endocrinology (19\%), pneumology (13\%), internal medicine (6\%), neonatology (5\%), etc.

Table 3 shows the distribution of patients enrolled in the BRO Biobank by disease type. The most representative diseases were rare diseases (56\%), followed by lung cancer (13\%), hematological neoplasms (4\%), and kidney transplantation (3\%). $25 \%$ of the patients had unknown diagnoses.

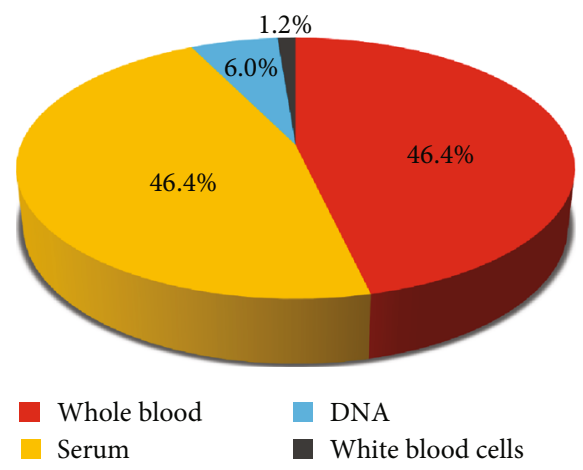

Figure 2: Distribution of biospecimens collected from healthy participants and banked in the BRO Biobank.

In July 2020, the BRO Biobank has banked approximately 751 biological samples from patients with various diseases. DNA samples account for $62 \%$ of the total number of samples stored in the BRO Biobank, followed by serum (10\%), plasma (9\%), FFPE tissue (46\%), white blood cells (5\%), RNA (5\%), frozen tissue (2\%), and urine (1\%) (Figure 4).

\subsection{Quality Control Assessment of the BRO Biobank. High-} throughput DNA and RNA analysis technologies (exome sequencing, RNA-seq, etc.) require high-quality nucleic acids. Therefore, one of the main challenges of biobanking is to ensure that stored samples are of high quality. Thus, we established several methods for sample quality control of the BRO Biobank and the procedure is described above in Materials and Methods. As an example, Table 4 shows the DNA quality assessment by measuring the ratio $\mathrm{OD}_{260} / \mathrm{OD}_{280}$ of some 
TABLE 2: Characteristics of patients enrolled in the BRO Biobank.

\begin{tabular}{lc}
\hline Patients' personal information & $N(\%)$ \\
\hline Gender $(N=433)$ & $173(40 \%)$ \\
Female & $260(60 \%)$ \\
Male & 11 years \\
\hline Age & 1 day-83 years \\
Median & \\
Range & $130(47 \%)$ \\
\hline Consanguinity $(N=274)$ & $144(53 \%)$ \\
Yes & $89(68 \%)$ \\
No & $26(20 \%)$ \\
\hline Degree of consanguinity $(N=130)$ & $15(12 \%)$ \\
$1^{\text {st }}$ degree & \\
$2^{\text {ed }}$ degree & $98(36 \%)$ \\
$3^{\text {rd }}$ degree & $175(64 \%)$ \\
\hline Family history of genetic diseases $(N=273)$ & $268(62 \%)$ \\
Yes & $165(38 \%)$ \\
\hline No & \\
\hline Purpose of enrollment $(N=433)$ & \\
Diagnosis and research & \\
Research only & \\
\hline
\end{tabular}

DNA samples of the BRO Biobank after at least 2 years of storage. The DNA quality assessment of all randomly selected BRO Biobank DNA samples is shown in Supplementary Materials Table S1. All samples showed a ratio between 1.8 and 2.1, confirming the absence of protein contamination and therefore the high purity of these DNA samples [27, 28]. Figure 5 shows the DNA integrity assessment of these samples by gel electrophoresis (Figure 5(a)), enzymatic digestion followed by gel electrophoresis (Figure 5(b)), and PCR (Figure 5(c)). Together, these results suggest that the DNA samples of the BRO Biobank are of good quality even after at least 2 years of storage.

As for DNA, RNA quality assessment was undertaken using several methods. Indeed, RNA samples of the BRO Biobank showed a ratio of 1.8 , confirming the absence of protein contamination and therefore the high purity of these samples (Table 4). The RNA quality assessment of all randomly selected BRO Biobank RNA samples is shown in Supplementary Materials Table S2. In addition, RNA integrity assessment of these samples by gel electrophoresis (Figure 6(a)) and RT-PCR (Figure 6(b)) showed that they have retained their integrity and therefore are well preserved after at least 2 years of storage.

\section{Discussion}

Biobanks have evolved into complex infrastructures, which significantly contribute to health research and operate at national, regional, and global levels $[35,36]$. Indeed, biobanks have accelerated the discovery and development of new treatments by providing sufficient and good-quality samples and data to translational medical research. There- fore, it is extremely important to set up a biobank in every medical research center. For this reason, we launched in November 2015 the BRO Biobank which is, to our knowledge, the first biobank in Morocco.

4.1. Impact of the BRO Biobank. There are different types of biobanks. Some biobanks collect samples donated by healthy donors from the general population. This type of biobank is named population-based biobanks and is an essential resource for studies exploring genetic and/or environmental factors implicated in the development of human diseases. These collections require the ability to follow the enrolled individuals over a long time to understand why some individuals develop certain diseases and other do not. China Kadoorie Biobank and UK Biobank are good examples of this type of biobank $[37,38]$. Other biobanks focus on a specific disease such as the French glioblastoma biobank, the $\beta$-thalassemia biobank, chronic kidney disease biobank, and lung cancer biobank, and these types of biobanks are called patient-based biobank or disease-oriented biobank $[5,7$, 39-55]. Other biobanks collect samples from patients with different diseases such as the Lausanne institutional biobank and the Japanese biobank $[56,57]$.

The BRO Biobank has samples from 2013 healthy donors who are representative of Eastern Morocco and can, therefore, be used to further our understanding of the factors that predispose this population to different diseases. Indeed, this population-based collection of the BRO Biobank will allow the long-term study and monitoring of healthy individuals in order to follow the natural development of various diseases in Eastern Morocco population. Furthermore, this cohort is very important in the frequency determination of carriers of autosomal recessive disorders, which are very common in Eastern Morocco due to the high prevalence of consanguineous marriage [58].

In addition to samples from healthy donors, the BRO Biobank harvests and stores samples from patients with various diseases. The majority of these samples are from patients with rare diseases. In fact, during the last five years, our Genetics Unit was the only laboratory in Eastern Morocco offering cytogenetic testing for constitutional chromosomal abnormalities and molecular testing for selected single-gene disorders resulting in availability of many research samples during patient's routine clinical care. Examples of this type of samples that were banked include hemoglobinopathies, syndromic intellectual disabilities, disorders of sex differentiation, and many other genetic conditions. It is worth noting that the rate of consanguineous marriages is still very high in Morocco and therefore, human genetic disorders are so widespread, but they remain uncharacterized $[59,60]$. Indeed, research resources in Morocco have been limited and thus, human genetic disorder studies face overwhelming challenges [61, 62]. Consequently, the BRO Biobank is of a great value because it offers unique advantages in studying consanguineous families and in identifying genes that are associated with human genetic disorders in Morocco. Identifying these genes could enable disease recognition and prevention, through genetic screening, which can greatly reduce disease severity as well as the impact and cost to society. 


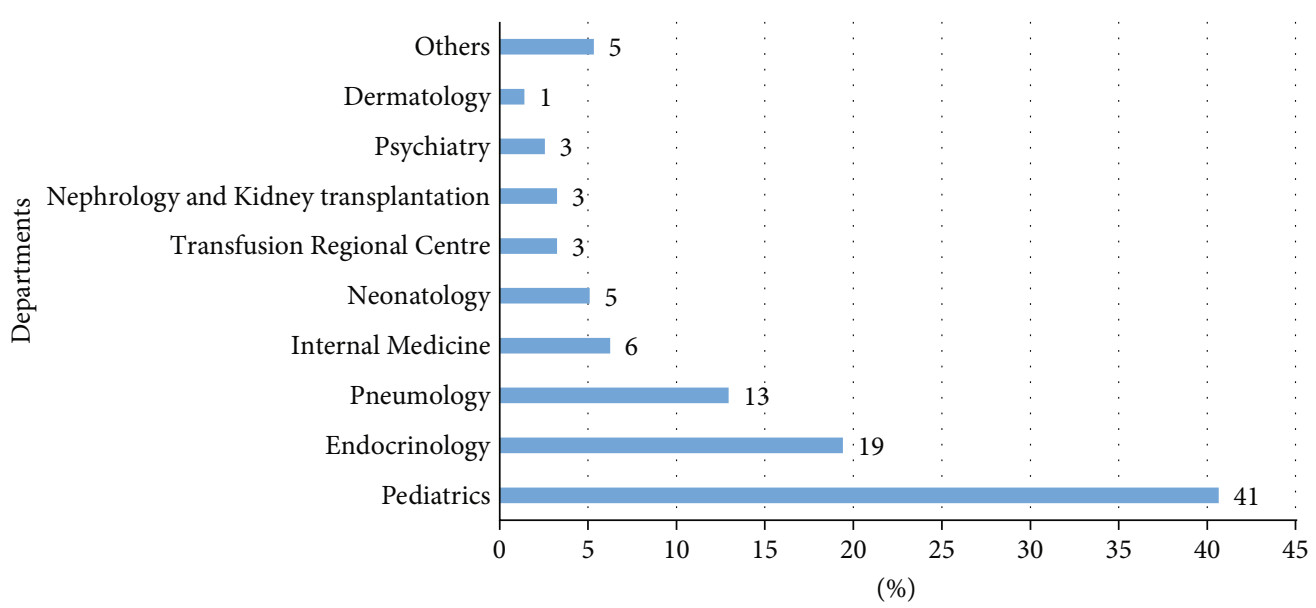

FIgURE 3: Distribution of biospecimens collected from healthy participants and banked in the BRO Biobank.

TABLE 3: Distribution of patients enrolled in the BRO Biobank by disease type.

\begin{tabular}{lc}
\hline Disease type & $N(\%)$ \\
\hline Rare diseases & $241(56 \%)$ \\
Hemoglobinopathy & $48(11 \%)$ \\
Intellectual disability & $47(11 \%)$ \\
Disorders of sex differentiation & $32(7 \%)$ \\
Myopathy & $30(7 \%)$ \\
Multiple endocrine neoplasia & $16(4 \%)$ \\
Genodermatosis & $11(3 \%)$ \\
Metabolic disease & $9(2 \%)$ \\
Autism spectrum disorders & $6(1 \%)$ \\
SMA & $5(1 \%)$ \\
Others & $37(9 \%)$ \\
Lung cancer & $56(13 \%)$ \\
Hematological neoplasms & $16(4 \%)$ \\
Kidney transplantation & $13(3 \%)$ \\
Unknown diagnosis & $107(25 \%)$ \\
\hline
\end{tabular}

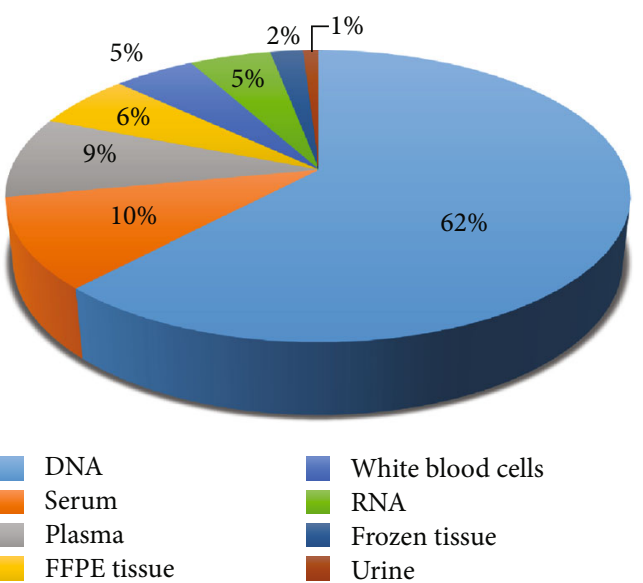

FIGURE 4: Distribution of collected biospecimens from patients banked by the BRO Biobank.
TABLE 4: Quality assessment of some DNA and RNA samples of the BRO Biobank after at least 2 years of storage using NanoDrop spectrophotometry.

\begin{tabular}{lcccccc}
\hline Samples & $\mathrm{D} 1$ & $\mathrm{D} 2$ & $\mathrm{D} 3$ & $\mathrm{R} 1$ & $\mathrm{R} 2$ & $\mathrm{R} 3$ \\
\hline Concentration $(\mathrm{ng} / \mu \mathrm{l})$ & 145.8 & 51 & 318.7 & 246.9 & 266 & 261.4 \\
$\mathrm{OD}_{260} / \mathrm{OD}_{280}$ & 1.91 & 1.99 & 1.96 & 1.8 & 1.8 & 1.8 \\
\hline
\end{tabular}

D1, D2, and D3: DNA samples; R1, R2, and R3: RNA samples.

In addition to samples from patients with rare diseases, the BRO Biobank collected samples from patients with various diseases such as kidney diseases, lung cancer, myeloproliferative neoplasms, and other conditions.

Together, the BRO Biobank is both a population-based biobank and a disease-oriented biobank. It is therefore a valuable resource for advancing personalized medicine in many diseases. It also offers the opportunity to study the causes of diseases affecting the population of Eastern Morocco.

4.2. Ethical and Legal Issues of the BRO Biobank. In Morocco, there is currently no specific regulation for the creation or activities of biobanks. Therefore, the BRO Biobank was established in accordance with regulations on biobanks already implemented in various countries as well as with the Moroccan law on the protection of individuals with regard to the processing of personal data [20-25]. Therefore, this work will help the creation of other biobanks in Morocco in accordance with ethical rules.

4.3. SOPs, Quality Control, and Data Management of the $B R O$ Biobank. Driven by the concern over research reproducibility, biobanks must follow standard operating procedures (SOPs) from sample collection to sample distribution. Thus, our first goal was to adopt good laboratory practices and a rigorous quality control system. Indeed, we have concentrated our effort on developing sample collection, processing, and storage procedures. Therefore, our team composed of highly trained molecular biologists was able to build standards and guidelines in accordance with SOPs that have been established by various biobank organizations, such as the International 


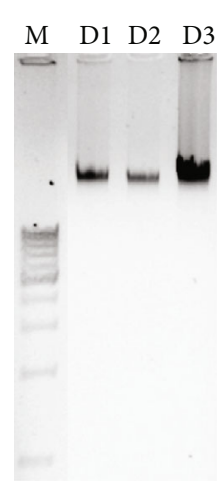

(a)

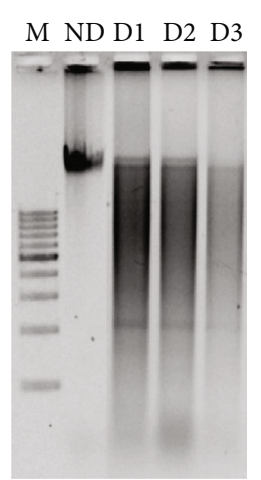

(b)

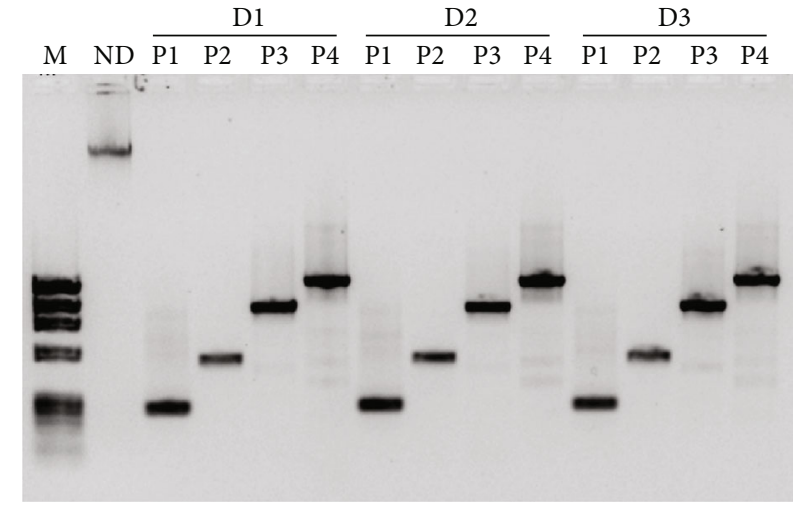

(c)

FIGURE 5: Quality assessment of DNA samples of the BRO Biobank after at least 2 years of storage: (a) $0.8 \%$ agarose gel electrophoresis of stored DNA samples; (b) $0.8 \%$ agarose gel electrophoresis of DNA samples digested with HindIII enzyme; (c) $1.2 \%$ agarose gel electrophoresis of PCR amplification of four regions of the $\beta$-globin gene using DNA samples of the BRO Biobank as templates. M: molecular weight marker (0.1-10 kb); D1, D2, and D3: DNA samples; ND: nondigested DNA; P1, P2, P3, and P4: regions of the $\beta$-globin gene amplified by PCR.

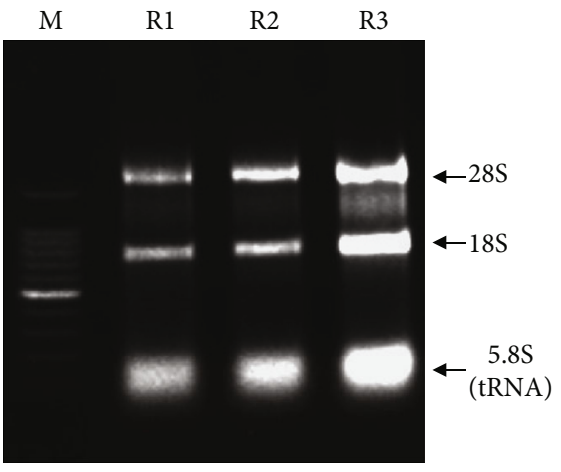

(a)

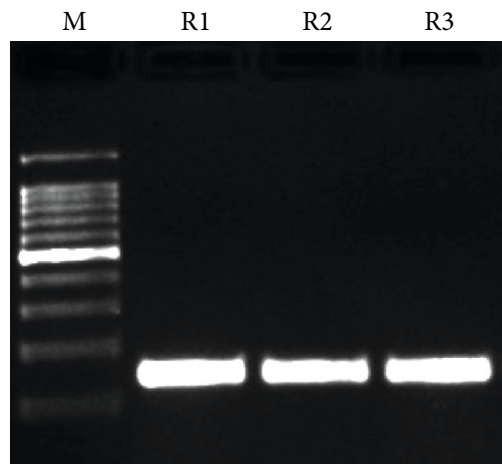

(b)

FIGURE 6: Quality assessment of RNA samples of the BRO Biobank after at least 2 years of storage: (a) 1.5\% nondenaturing agarose gel electrophoresis of RNA samples; (b) 2.5\% agarose gel electrophoresis of RT-PCR amplification of a region of GAPDH gene using RNA samples of the BRO Biobank as templates. M: molecular weight marker (0.1-10 kb); R1, R2, and R3: RNA samples.

Society for Biological and Environmental Repositories [9-15]. However, so far, our SOPs have not had any accreditation or certification from any biobanking accreditation system. Therefore, our next step is to achieve accreditation for the BRO Biobank by an official accreditation body. In fact, the granting of accreditation will give confidence to users of biobank samples and facilitate the increasing interinstitutional use of research materials from biobanks [63].

Sample quality control is another very important concern of biobanking. Therefore, we have established several methods for sample quality control of the BRO Biobank in accordance with international guidelines on biobanking [64-68]. DNA integrity following extraction from blood and tissue samples was evaluated, before and after long storage, by gel electrophoresis, enzymatic digestion, and PCR. Similarly, RNA integrity was evaluated by gel electrophoresis and RT-PCR. Furthermore, purity of DNA and RNA isolated from different biospecimens was determined using NanoDrop spectrophotometry. Overall, our results suggested that samples of the BRO Biobank are of high quality and therefore suitable for high-throughput nucleic acid analysis.

In addition to collecting and storing samples, biobanking also involves collecting, managing, and protecting patients' personal and clinical data. Indeed, biobanks require IT and data management systems for tracking and monitoring sample locations as well as for managing patients' personal information [14, 19]. However, due to lack of resources, data management of patients' personal information and samples' location in the BRO Biobank were done simply using Microsoft Excel. Therefore, it is extremely important to acquire IT and data management systems for the BRO Biobank, as the number of samples is increasing and data management is becoming more and more complicated.

4.4. Challenges of the BRO Biobank. The major challenge of the BRO Biobank is the involvement of physicians and surgeons in the biobanking process. Indeed, health professionals play a key role in the recruitment of patients into biobanks. 
We have recently assessed the knowledge and attitude of health professionals in Eastern Morocco towards biobanks and their willingness to recruit patients into biobanks. We found that our health professionals showed a notable lack of knowledge about biobanks. However, the majority were willing to donate their own biospecimens and supported the recruitment of patients into biobanks [69]. We have also evaluated the knowledge of our patients and their attitudes towards biobanks and the reasons that motivate them to participate in biobanks. We found that the majority of our patient expressed their willingness to participate in biobanking through donation of biospecimens associated with personnel and health data [70]. These studies were good opportunities to raise awareness among health professionals and patients about the interest of the BRO Biobank in the development of biomedical research in Eastern Morocco.

Another major challenge we encountered during biobanking was complete data collection of the patient's demographic and clinical information. This task also depends on the involvement of physicians and surgeons in the biobanking process. Therefore, a big effort is needed to raise awareness among health professionals about the importance of complete data from patients to the success of biobanking. In addition, special measures need to be taken for the data collection of the BRO Biobank, which can in fact be integrated into the patient's routine clinical care.

\section{Conclusion}

The BRO Biobank is the largest sample collection in Morocco and is ready to distribute samples to national and international researchers. Therefore, the BRO Biobank is a valuable resource for advancing translational research and personalized precision medicine in North Africa.

\section{Abbreviations}

BRO Biobank: Eastern Morocco biobank

FFPE: $\quad$ Formalin-fixed, paraffin-embedded

PBS: $\quad$ Phosphate-buffered saline

RNAlater: RNA-stabilizing buffer

SOP: $\quad$ Standard operating procedure

TE buffer: Tris-EDTA buffer

WBC: White blood cells

\section{Data Availability}

Researchers wishing to use the clinical data and/or biological materials of the BRO Biobank have to complete a request form. If their request is accepted by the scientific board, a material transfer agreement is signed and the data and biological materials can be supplied.

\section{Conflicts of Interest}

The authors declare that they have no conflicts of interest.

\section{Authors' Contributions}

SL, RB, MO, and MB were responsible for the biobank design. All authors were responsible for the implementation of the biobank. SL, Mounia EE, and Manal EE were responsible for data management. SL, KYB, IB, Mounia EE, Manal $\mathrm{EE}, \mathrm{RB}, \mathrm{MT}, \mathrm{MC}, \mathrm{MO}$, and $\mathrm{MB}$ were responsible for the analysis and interpretation of the data. SL, RB, MC, MO, and MB were responsible for the writing of the manuscript.

\section{Acknowledgments}

We are grateful to all patients at the participating health centers and to all blood donors. We thank the president of the University Mohammed Premier, Pr. Yassine Zarhloule, for his valuable support. We also thank Pr. Abderrahim Azzouzi and all the administrative staff of the Faculty of Medicine and Pharmacy of Oujda for their valuable support and encouragement throughout the entire work. We also thank the director of the University Hospital Center Mohammed VI Pr. Abdelkarim Daoudi and the Regional Director of the Ministry of Health in Eastern Morocco Dr. Koualla for their support. We thank Dr. S. Mentak and Dr. Mouad Edderkaoui for proofreading the manuscript.

\section{Supplementary Materials}

Table S1: quality assessment of DNA samples of the BRO Biobank using NanoDrop spectrophotometry. Table S2: quality assessment of RNA samples of the BRO Biobank using NanoDrop spectrophotometry. (Supplementary Materials)

\section{References}

[1] J. Kinkorova and O. Topolcan, "Biobanks European infrastructure," Casopís Lékař̀ Českých, vol. 155, no. 3, pp. 4446, 2016.

[2] S. Al Diffalha, K. C. Sexton, P. H. Watson, and W. E. Grizzle, "The importance of human tissue bioresources in advancing biomedical research," Biopreservation and Biobanking, vol. 17, no. 3, pp. 209-212, 2019.

[3] C. Compton and A. Kelly, "Biospecimen banking in the postgenome era," Genomic and Personalized Medicine: V1-2, vol. 1, pp. 229-236, 2013.

[4] P. Hofer-Picout, H. Pichler, J. Eder et al., "Conception and implementation of an Austrian biobank directory integration framework," Biopreservation and Biobanking, vol. 15, no. 4, pp. 332-340, 2017.

[5] K. Washetine, S. Heeke, C. Bonnetaud et al., "Establishing a dedicated lung cancer biobank at the University Center Hospital of Nice (France). Why and how?," Cancers (Basel), vol. 10, no. 7 , p. $220,2018$.

[6] M. Conroy, J. Sellors, M. Effingham et al., "The advantages of UK Biobank's open-access strategy for health research," Journal of Internal Medicine, vol. 286, no. 4, pp. 389-397, 2019.

[7] T. N. Haregu, S. Nanayakkara, B. Kingwell et al., "The Baker Biobank: understanding cardiovascular outcomes," Heart, Lung and Circulation, vol. 28, p. S191, 2019. 
[8] L. Coppola, A. Cianflone, A. M. Grimaldi et al., "Biobanking in health care: evolution and future directions," Journal of Translational Medicine, vol. 17, no. 1, p. 172, 2019.

[9] F. Betsou, R. Barnes, T. Burke et al., "Human biospecimen research: experimental protocol and quality control tools," Cancer Epidemiology, Biomarkers \& Prevention, vol. 18, no. 4, pp. 1017-1025, 2009.

[10] "Organization for Economic Cooperation and Development (OECD) guidelines for human biobanks and human genetic databases," 2009.

[11] J. Vaught and N. C. Lockhart, "The evolution of biobanking best practices," Clinica Chimica Acta, vol. 413, no. 19-20, pp. 1569-1575, 2012.

[12] "NCI Best Practices for Biospecimen Resources," 2016.

[13] L. D. Campbell, J. J. Astrin, Y. DeSouza et al., "The 2018 revision of the ISBER best practices: summary of changes and the editorial team's development process," Biopreservation and Biobanking, vol. 16, no. 1, pp. 3-6, 2018.

[14] W. Paskal, A. M. Paskal, T. Dębski, M. Gryziak, and J. Jaworowski, "Aspects of modern biobank activity-comprehensive review," Pathology Oncology Research, vol. 24, no. 4, pp. 771-785, 2018.

[15] "European, Middle Eastern \& African Society for Biopreservation and Biobanking (ESBB): Vision, Mission \& Goals," 2020.

[16] M. Mendy, E. Caboux, B. S. Sylla, J. Dillner, J. Chinquee, and C. Wild, "Infrastructure and facilities for human biobanking in low- and middle-income countries: a situation analysis," Pathobiology, vol. 81, no. 5-6, pp. 252-260, 2014.

[17] R. M. Labib, M. M. Mostafa, A. S. Alfaar et al., "Biorepository for pediatric cancer with minimal resources: meeting the challenges," Biopreservation and Biobanking, vol. 14, no. 1, pp. 916,2016

[18] M. H. Zawati, A. M. Tassé, M. Mendy, E. Caboux, M. Lang, and on Behalf of Biobank and Cohort Building Network Members, "Barriers and opportunities in consent and access procedures in low- and middle-income country biobanks: meeting notes from the BCNet training and general assembly," Biopreservation and Biobanking, vol. 16, no. 3, pp. 171-178, 2018.

[19] J. Fachiroh, E. K. Dwianingsih, A. E. Wahdi et al., "Development of a biobank from a legacy collection in Universitas Gadjah Mada, Indonesia: proposed approach for centralized biobank development in low-resource institutions," Biopreservation and Biobanking, vol. 17, no. 5, pp. 387-394, 2019.

[20] "Bullten officiel. Loi n 09-08 du 18 février 2009 relative à la protection des personnes physiques à l'égard du traitement des données à caractère personnel," July 2020, https://www .dgssi.gov.ma/sites/default/files/attached_files/loi_0908protection_donnees_personnelles.pdf.

[21] M. Belge, "Loi du 19 décembre 2008 relative à l'obtention et à l'utilisation de matériel corporel humain destiné à des applications médicales humaines ou à des fins de recherche scientifique," July 2020, http://www.ipg.be/wp-content/uploads/doc/ Loi\%202008\%2012\%2019\%20mat\%C3\%A9riel\%20corporel\% 20humain).pdf.

[22] D. Ethikrat, "Les biobanques humaines destinées à la recherche," July 2020, https://www.ethikrat.org/fileadmin/ Publikationen/Stellungnahmen/franzoesisch/DER_StnBiob_ Frz_mitKennwort.pdf.

[23] Legifrance, "Loi $n^{\circ}$ 2012-300 du 5 mars 2012 relative aux recherches impliquant la personne humaine," July 2020,
https://www.legifrance.gouv.fr/affichTexte.do?cidTexte= JORFTEXT000025441587\&categorieLien=id.

[24] Ministry of Social Affairs and Health Finland, "Biobank Act," July 2020, https://www.finlex.fi/fi/laki/kaannokset/2012/ en20120688.pdf.

[25] Ministry of Welfare, "The Biobanks and Health Databanks Act," July 2020, https://www.government.is/media/ velferdarraduneyti-media/media/acrobat-enskar_sidur/ Biobanks-Act-as-amended-2015.pdf.

[26] M. Ghaheri, D. Kahrizi, K. Yari, A. Babaie, R. S. Suthar, and E. Kazemi, "A comparative evaluation of four DNA extraction protocols from whole blood sample," Cellular and Molecular Biology (Noisy-le-Grand, France), vol. 62, no. 3, pp. 120-124, 2016.

[27] C. le Page, M. Köbel, M. de Ladurantaye et al., "Specimen quality evaluation in Canadian biobanks participating in the COEUR repository," Biopreservation and Biobanking, vol. 11, no. 2, pp. 83-93, 2013.

[28] L. Craciun, S. A. Spinette, M. Rassy et al., "Tumor banks: a quality control scheme proposal," Frontiers in Medicine(Lausanne), vol. 6, p. 225, 2019.

[29] Network CTR, “Assessing quality of nucleic acids," 2008, July 2020, https://www.google.com/search?q=pnf-rcbt-5.1.002evaluation-de-la-qualite-des-acides-nucleiques.pdf\%3E\&oq= pnf-rcbt-5.1.002-evaluation-de-la-qualite-des-acidesnucleiques.pdf\%3E\&aqs $=$ chrome..69i57.913j0j9\&sourceid $=$ chrome\&ie $=$ UTF- 8 .

[30] J. Ortega-Pinazo, T. Diaz, B. Martinez, A. Jimenez, M. J. PintoMedel, and P. Ferro, "Quality assessment on the long-term cryopreservation and nucleic acids extraction processes implemented in the andalusian public biobank," Cell and Tissue Banking, vol. 20, no. 2, pp. 255-265, 2019.

[31] L. Calleros-Basilio, M. A. Cortés, A. García-Jerez et al., "Quality assurance of samples and processes in the Spanish Renal Research Network (REDinREN) Biobank," Biopreservation and Biobanking, vol. 14, no. 6, pp. 499-510, 2016.

[32] J. Botling, K. Edlund, U. Segersten et al., "Impact of thawing on RNA integrity and gene expression analysis in fresh frozen tissue," Diagnostic Molecular Pathology, vol. 18, no. 1, pp. 44-52, 2009.

[33] "Regional map of Morocco following the administrative division of 2015," July 2020, https://commons.wikimedia.org/ wiki/File:Regional_map_of_Morocco_-_post_2015.svg.

[34] “Morocco map location," July 2020, https://maps-morocco .com/morocco-map-location.

[35] M. T. Mayrhofer and B. Prainsack, "Being a member of the club: the transnational (self-) governance of networks of biobanks," International Journal of Risk Assessment and Management, vol. 12, no. 1, pp. 64-81, 2009.

[36] J. R. Harris, P. Burton, B. M. Knoppers et al., "Toward a roadmap in global biobanking for health," European Journal of Human Genetics : EJHG, vol. 20, no. 11, pp. 1105-1111, 2012.

[37] Z. Chen, J. Chen, R. Collins et al., "China Kadoorie Biobank of 0.5 million people: survey methods, baseline characteristics and long-term follow-up," International Journal of Epidemiology, vol. 40, no. 6, pp. 1652-1666, 2011.

[38] G. Watts, UK Biobank opens its data vaults to researchers, vol. 344, no. mar30 2, 2012British Medical Journal Publishing Group, 2012.

[39] G. Thomas, E. Williams, and NISCTB motSPPot, "Chernobyl thyroid tumor bank," The Journal of Clinical Endocrinology \& Metabolism, vol. 86, no. 3, p. 1428, 2001. 
[40] T. Y. Chu, K. S. Hwang, M. H. Yu, H. S. Lee, H. C. Lai, and J. Y. Liu, "A research-based tumor tissue bank of gynecologic oncology: characteristics of nucleic acids extracted from normal and tumor tissues from different sites," International Journal of Gynecological Cancer, vol. 12, no. 2, pp. 171-176, 2002.

[41] A. A. Patel, A. Kajdacsy-Balla, J. J. Berman et al., "The development of common data elements for a multi-institute prostate cancer tissue bank: the Cooperative Prostate Cancer Tissue Resource (CPCTR) experience," BMC Cancer, vol. 5, no. 1, p. 108, 2005.

[42] H. Christensen, J. S. Nielsen, K. M. Sorensen, M. Melbye, and I. Brandslund, "New national biobank of the Danish Center for Strategic Research on type 2 diabetes (DD2)," Clinical Epidemiology, vol. 4, pp. 37-42, 2012.

[43] K. Majidzadeh-A, A. Kaviani, R. Esmaeili et al., "Iranian Breast Cancer Bio-Bank: the activity and challenging issues," Cell and Tissue Banking, vol. 14, no. 1, pp. 11-20, 2013.

[44] B. Stengel, C. Combe, C. Jacquelinet et al., "The French chronic kidney disease-renal epidemiology and information network (CKD-REIN) cohort study," Nephrology Dialysis Transplantation, vol. 29, no. 8, pp. 1500-1507, 2013.

[45] M. Ahram, R. Zaza, L. Ibayyan et al., "Towards establishing a multiple sclerosis biobank in Jordan," The International Journal of Neuroscience, vol. 124, no. 11, pp. 812-817, 2014.

[46] G. J. Navis, P. J. Blankestijn, J. Deegens et al., "The Biobank of nephrological diseases in the Netherlands cohort: the String of Pearls Initiative collaboration on chronic kidney disease in the university medical centers in the Netherlands," Nephrology, Dialysis, Transplantation, vol. 29, no. 6, pp. 1145-1150, 2014.

[47] K. Yu, J. Zhang, X. Li et al., "Establishment and management of a lung cancer biobank in Eastern China," Thorac Cancer, vol. 6, no. 1, pp. 58-63, 2015.

[48] C. Baldo, V. Viotti, E. Maioli et al., "Galliera Genetic Bank: a DNA and cell line biobank from patients affected by genetic diseases," Open Journal of Bioresources, vol. 3, no. 1, 2016.

[49] L. C. Cosenza, L. Breda, G. Breveglieri et al., "A validated cellular biobank for $\beta$-thalassemia," Journal of Translational Medicine, vol. 14, no. 1, article 255, 2016.

[50] A. S. El Obeid and I. Al Abdudlkarim, "The role of biobanks in elucidating prevalent genetic diseases in Saudi Arabia," Drug Discoveries \& Therapeutics, vol. 10, no. 4, pp. 226-233, 2016.

[51] Y. R. Rubinstein, M. Posada de la Paz, and M. Mora, "Rare disease biospecimens and patient registries: interoperability for research promotion, a European example: EuroBioBank and SpainRDR-BioNER," Advances in Experimental Medicine and Biology, vol. 1031, pp. 141-147, 2017.

[52] M. Tada, M. Hirata, M. Sasaki et al., "The Rare Disease Bank of Japan: establishment, current status and future challenges," Human Cell, vol. 31, no. 3, pp. 183-188, 2018.

[53] FGB network, A. Clavreul, G. Soulard et al., "The French glioblastoma biobank (FGB): a national clinicobiological database," Journal of Translational Medicine, vol. 17, no. 1, p. 133, 2019.

[54] O. F. Khabour and A. Abu-Siniyeh, "Challenges that face the establishment of diabetes biobank in Jordan: a qualitative analysis of an online discussion forum," Journal of Multidisciplinary Healthcare, vol. 12, pp. 229-234, 2019.

[55] R. Wagner, L. Ayoub, S. Kahnamoui et al., "Establishment of a biobank for human lung tissues of congenital diaphragmatic hernia and congenital pulmonary airway malformation," Journal of Pediatric Surgery, vol. 54, no. 11, pp. 2439-2442, 2019.
[56] V. Mooser and C. Currat, "The Lausanne Institutional Biobank: a new resource to catalyse research in personalised medicine and pharmaceutical sciences," Swiss Medical Weekly, vol. 144, article w14033, 2014.

[57] A. Nagai, M. Hirata, Y. Kamatani et al., "Overview of the BioBank Japan Project: study design and profile," Journal of Epidemiology, vol. 27, no. 3, pp. S2-s8, 2017.

[58] M. El Ouardani, L. Habibeddine, A. Bouali, N. Boukhatem, J. Talbi, and H. El Ossmani, "Etude de la consanguinite dans la region du Maroc Oriental," Aminnovresappisci, vol. 9, no. 2, pp. 148-152, 2019.

[59] J. Talbi, A. Khadmaoui, A. Soulaymani, and A. Chafik, "Etude de la consanguinité dans la population marocaine. Impact sur le profil de la santé," Antropo, vol. 15, pp. 1-11, 2007.

[60] I. C. Jaouad, S. C. Elalaoui, A. Sbiti, F. Elkerh, L. Belmahi, and A. Sefiani, "Consanguineous marriages in Morocco and the consequence for the incidence of autosomal recessive disorders," Journal of Biosocial Science, vol. 41, no. 5, pp. 575-581, 2009.

[61] M. el-Azami-el-Idrissi, M. Lakhdar-Idrissi, K. Ouldim et al., "Improving medical research in the Arab world," The Lancet, vol. 382, no. 9910, pp. 2066-2067, 2013.

[62] K. Belhassan, K. Ouldim, and A. A. Sefiani, "Genetics and genomic medicine in Morocco: the present hope can make the future bright," Molecular Genetics \& Genomic Medicine, vol. 4, no. 6, pp. 588-598, 2016.

[63] F. Betsou, "Quality assurance and quality control in biobanking," in Biobanking of Human Biospecimens, pp. 23-49, Springer, 2017.

[64] S. Imbeaud, E. Graudens, V. Boulanger et al., "Towards standardization of RNA quality assessment using userindependent classifiers of microcapillary electrophoresis traces," Nucleic Acids Research, vol. 33, no. 6, p. e56, 2005.

[65] S. Fleige, V. Walf, S. Huch, C. Prgomet, J. Sehm, and M. W. Pfaffl, "Comparison of relative mRNA quantification models and the impact of RNA integrity in quantitative real-time RT-PCR," Biotechnology Letters, vol. 28, no. 19, pp. 16011613, 2006.

[66] S.-M. Shim, J.-H. Kim, S.-E. Jung et al., "Multilaboratory assessment of variations in spectrophotometry-based DNA quantity and purity indexes," Biopreservation and Biobanking, vol. 8, no. 4, pp. 187-192, 2010.

[67] J. Glasel, "Validity of nucleic acid purities monitored by $260 \mathrm{~nm} / 280 \mathrm{~nm}$ absorbance ratios," BioTechniques, vol. 18, no. 1, pp. 62-63, 1995.

[68] S. D. Jewell, M. Srinivasan, L. M. McCart et al., "Analysis of the molecular quality of human tissues: an experience from the Cooperative Human Tissue Network," American Journal of Clinical Pathology, vol. 118, no. 5, pp. 733-741, 2002.

[69] S. Lhousni, R. Boulouiz, N. Abda, M. Tajir, M. Bellaoui, and M. Ouarzane, "Assessment of knowledge, attitudes and support of health professionals towards biobanks in Eastern Morocco," Open Journal of Epidemiology, vol. 9, no. 3, pp. 191-201, 2019.

[70] S. Lhousni, F. Daoudi, I. Belmokhtar et al., "Patients' knowledge and attitude toward biobanks in Eastern Morocco," Biopreservation and Biobanking, vol. 18, no. 3, pp. 189-195, 2020. 